\title{
Erratum: F-theory and the classification of little strings [Phys. Rev. D 93, 086002 (2016)]
}

\author{
Lakshya Bhardwaj, Michele Del Zotto, Jonathan J. Heckman, David R. Morrison, \\ Tom Rudelius, and Cumrun Vafa
}

(Received 30 May 2019; published 9 July 2019)

DOI: 10.1103/PhysRevD.100.029901

This erratum concerns the examples of little string theories discussed between Eqs. (7.4) and (7.5). We reproduce these examples below:

$$
\begin{aligned}
& \mathfrak{s} \mathfrak{o}(7) \quad \mathfrak{s} \mathfrak{u}(4) \\
& \underset{[\operatorname{Sp}(1)]}{2} \| 2 \\
& \mathfrak{s} \mathfrak{o}(8) \quad \mathfrak{s} \mathfrak{t}(4) \\
& \underset{[\operatorname{Sp}(2)]}{2} \| 2 \\
& \mathfrak{g}_{2} \quad \mathfrak{s} \mathfrak{i t}(4) \\
& 2|| 2 \\
& \mathfrak{s} \mathfrak{o}(12) \quad \mathfrak{g} \mathfrak{i t}(6) \\
& \underset{\left[N_{s}=1\right]}{2} \| 2 \\
& \underset{2}{\mathfrak{s} \mathfrak{o}(13)} \|^{\mathfrak{j} \mathfrak{t}(7)}
\end{aligned}
$$

These theories cannot be constructed using methods detailed in the original manuscript. The reason for this is that the $\mathfrak{g} \mathfrak{t}(N)$ for $N=4,6,7$ appearing in the examples above is constructed by an $I_{N}$ singularity on a -2 curve. But an $I_{N}$ singularity on a -2 curve cannot consistently intersect an $I_{M}^{*}$ type singularity required to construct $\mathfrak{S} \mathfrak{o}(7), \mathfrak{g} \mathfrak{v}(8), \mathfrak{S} \mathfrak{v}(12), \mathfrak{S} \mathfrak{o}(13), \mathfrak{g}_{2}$ in the examples above. In more detail, the order of vanishing of $f$ for an $I_{N}$ singularity is zero. If the $I_{N}$ singularity wraps a -2 curve $C$ in the base $B$, then the number of points on $C$ where $f$ vanishes is computed as $-4 K_{B} \cdot C=0$ where $K_{B}$ is the canonical divisor on $B$. This is in contradiction with the fact that $C$ intersects another curve $D$ carrying an $I_{M}^{*}$ singularity on which $f$ vanishes at least to order two.

The above examples should thus be placed in Sec. 10 of the original manuscript where we discuss examples of little string theories (and stands for superconformal field theories) that we expect to be constructed by some nongeometric phase of string theory. Indeed, the construction for the above examples was recently provided in [1] using the frozen phase of Ftheory which is a nongeometric phase of F-theory.

Related to the above examples, there is an error in Appendix B where we discuss Weierstrass models for the above examples. The erroneous statements start from the beginning of the paragraph containing Eq. (B.3) "We now consider the Kodaira type III base configuration, which ..." to the end of Appendix B “... on two tangent -2 curves discussed in Section VII."

[1] L. Bhardwaj, Revisiting the classifications of $6 d$ SCFTs and LSTs, arXiv:1903.10503. 